\title{
Parameter Values of Android APIs: A Preliminary Study on 100,000 Apps
}

\author{
Li Li, Tegawendé F. Bissyandé, Jacques Klein, Yves Le Traon \\ Interdisciplinary Centre for Security, Reliability and Trust, University of Luxembourg, Luxembourg \\ \{li.li, tegawende.bissyande, jacques.klein, yves.letraon\}uni.lu
}

\begin{abstract}
Parameter values are important elements for understanding how Application Programming Interfaces (APIs) are used in practice. In the context of Android, a few number of API methods are used pervasively by millions of apps, where these API methods provide app core functionality. In this paper, we present preliminary insights from ParamHarver, a purely static analysis approach for automatically extracting parameter values from Android apps. Investigations on 100,000 apps illustrate how an in-depth study of parameter values can be leveraged in various scenarios (e.g., to recommend relevant parameter values, or even, to some extent, to identify malicious apps).
\end{abstract}

\section{INTRODUCTION}

In recent years, we have been observing the spreading of Android support for numerous devices, varying from small devices such as smart phones and watches to large devices such as fridges, TV sets, etc. This momentum of Android is accompanied by the development community who produces large numbers of apps - 1,6 million apps as of July 2015 that users leverage in all activities of their daily life (from email and social networking to text processing and banking operations).

Unfortunately, the high market share of Android has contributed to making him a target of choice for malware writers. For example, a number of malicious apps have harmed users' finances by sending premium SMS messages with payments being collected by malware writers. In such cases, however, an analyst could easily prevent the malicious behaviour if he could check the value phone number beforehand (e.g., by extracting the value of the first parameter of SMS-sending API method sendTextMessage). To allow a more systematic check by any app analyst, one solution could be to build a blacklist of premium numbers by collecting, based on a large set of Android apps, all constant values that are passed to the sendTextMessage API method.

A recent study with the Harvester [11] approach has demonstrated that sensitive runtime data from Android apps can be used to identify malware. With the Bouncer, Google Play's maintainers are already leveraging runtime data to filter out those apps that violate the store's policy (e.g., sending SMS to their blacklisted phone numbers). Such sensitive data often involve parameter values for some sensitive Android API methods.

We argue that, besides malicious app identification, a harvest of parameter values can be leveraged to support other analyses. Consider for example an API method $m$, and the set $s$ of parameter values that $m$ uses. If $s$ is collected from a large enough dataset of apps, one should be able, based on $s$, to puzzle out what are the standard uses of $m$ in practice. Thus, building on the harvested values, one can for instance suggest appropriate parameter values to developers who would want to use $m$ in the future. This recommendation is often essential in cases where proper documentation is lacking. As an example, let us consider API method getLastKnownLocation(provider) which takes a string parameter named ("provider"). In the official documentation 1 , the explanation for the parameter value simply states: "provider: the name of the provider", which may be insufficient for guiding developers.

In this paper, we aim to investigate to what extent parameter values can be harvested and leveraged in Android analyses. To fulfil this endeavour, we first need to extract all possible parameter values of a given set of API methods. Unfortunately, state-of-the-art works such as Google Bouncer and Harvester are not publicly available. Furthermore, both the Bouncer and Harvester are dynamic and thus require runtime execution of apps. Such approaches may not scale to app market sizes, and may also be bypassed by sophisticated malware (e.g., such malware often perform malicious behaviour at a specific time and can even hide their behavior when they detect a testing environment).

To mitigate the limitations of dynamic analysis, and to scale our investigations, we propose an approach called ParamHarver for harvesting API parameter values purely statically. ParamHarver performs backward string analysis to extract the possible parameter values of a given API method. Overall, we make the following contributions:

- a static analysis tool called ParamHarver that collects preset (i.e., hard-coded constant) parameter values for a given set of Android API methods.

- an evaluation of ParamHarver's usability for a set of 100,000 Android apps.

- a preliminary study on the parameter values reported by the experiments performed on the dataset of 100,000 Android apps.

\section{EXtRACTION OF PARAMETER VAlueS}

To motivate our work on ParamHarver, we discuss a realworld example in Section II-A Subsequently, in Section II-B we discuss the details for implementing ParamHarver, which

\footnotetext{
${ }^{1}$ http://developer.android.com/reference/android/location/Location Manager.html\#getLastKnownLocation(java.lang.String)
} 


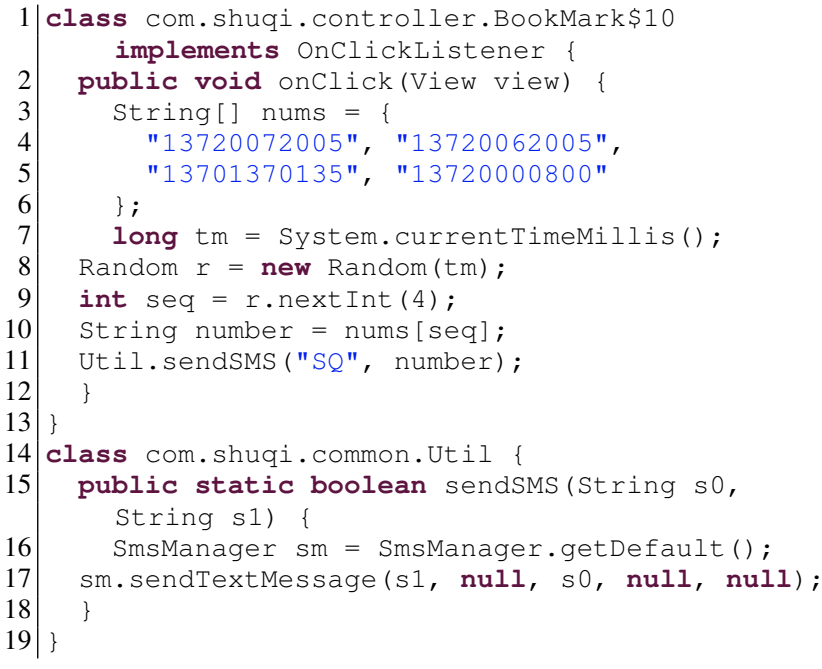

Listing 1: A code snippet extracted from a real-world Android app whose package name is com.shuqi.controller. Some irrelevant code are excluded for simplicity reasons.

in summary performs inter-procedural string analysis to collect parameter values of Android API methods.

\section{A. Motivating Example}

Listing 11 shows a code snippe ${ }^{2}$ taken from a real-world Android app called com.shuqi.controller. This snippet sends a text message through the Android API sendTextMessage, which include two relevant string arguments: $s l$ and $s O$ (line 17). In order to extract their values, backward string analysis is needed. Furthermore, both $s 1$ and $s O$ are parameters of sendSMS (line 15), and are actually defined in method onClick (line 2). Thus, the backward string analysis must also be inter-procedural.

As shown in lines 3-6 within onClick(), there are actually four (premium) phone numbers defined in the array nums. In other words, these four numbers are possible parameter values of sendTextMessage(). If these numbers are successfully harvested, we could use them later on to infer whether the investigated app is likely malicious or not by simply comparing them with a set of known premium numbers (or vulnerable numbers).

\section{B. Implementation}

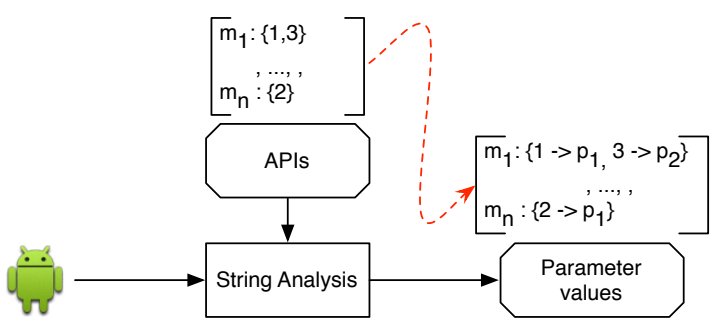

Fig. 1: The main working process of ParamHarver.

\footnotetext{
${ }^{2}$ Note that we have rewritten this snippet to Java source code in order to ease the understanding.
}

Fig. 1]illustrates the main working process of ParamHarver, which takes as inputs an Android app and a set of Android API methods along with their meta-data (configured by users of ParamHarver) which indicate precisely the position of interested string parameters that ParamHarver tracks. As an example, the meta-data for method sendTextMessage() in Listing 1 is sendTextMessage : 1, 3, requesting ParamHarver to harvest the value of the first and third parameters of sendTextMessage(). ParamHarver performs backward string analysis for every concerned parameters and outputs their values. The backward tracking will keep iterating until 1) the parameter value is localized or 2) the root of the controlflow graph (CFG) is reached, as it is the case for dynamically generated values. If the first constraint is met, ParamHarver simply outputs the identified values. Otherwise, the second constraint is met, indicating that the parameter value is not correctly extracted. In this case, ParamHarver returns a regular expression $\left(.^{*}\right)$, showing that the parameter value could be potentially everything. Existing works such as the one presented by Octeau et al. [9] can be leveraged to mitigate this.

ParamHarver is implemented on top of COAL, a constant propagation language and solver [10]. Because Android apps do not have a single entry-point (e.g., main() in Java apps), ParamHarver artificially builds a dummy main method that takes into account both lifecycle and callback methods, following our previous experiences [2], [4].

\section{EMPIRICAL INVESTIGATION}

In this section, we evaluate ParamHarver empirically on a set of 100,000 real-world Android apps, in an attempt to address the following research questions:

- RQ1: What is the distribution of parameter values of Android API methods.

- RQ2: Is it feasible to recommend possible parameter values for a given Android API method?

- RQ3: Is it feasible to flag malicious apps based on the harvested parameter values?

- RQ4: Is it feasible to perform trend analysis on the harvested parameter values?

\section{A. Experimental Setup}

As introduced in the previous section, ParamHarver takes as inputs two artifacts: Android Apps and API methods.

Android Apps. We randomly select 100,000 Android malicious apps from a data set of over 2 million apps previously collected from Google Play and other third-party markets (e.g., appchina, anzhi, etc.) [1], [3]. In this study, we consider an Android app to be malicious if at least five anti-virus products hosted on VirusTota ${ }^{3}$ have flagged it as such.

Android API methods. In this experiment, for simplicity reasons (i.e., to reduce execution time, as well as manual evaluation efforts), we select the top 10 most used Android API methods for our preliminary investigation. In order to

\footnotetext{
${ }^{3}$ https://www.virustotal.com: A free online virus, malware and url scanner that hosts over 50 anti-virus products including AVG, McAfee, et al.
} 
collect these top 10 API methods, we first performed a quick study based on the sampled set of 100,000 apps. The study results are shown in Table II Note that in this study we only consider such API methods that have at least one string parameter.

TABLE I: The top 10 used Android API methods (with taking at least one string parameter).

\begin{tabular}{lllc}
\hline Seq & Method Name & Class Name & Count \\
\hline 1 & getLastKnownLocation & LocationManager & 40,609 \\
2 & requestLocationUpdates & LocationManager & 18,070 \\
3 & isProviderEnabled & LocationManager & 8,105 \\
4 & putString & Settings\$System & 3,746 \\
5 & sendTextMessage & SmsManager & 3,414 \\
6 & getAccountsByType & AccountManager & 2,089 \\
7 & putInt & Settings\$System & 1,666 \\
8 & restartPackage & ActivityManager & 1,272 \\
9 & setVideoPath & VideoView & 956 \\
10 & getProvider & LocationManager & 922 \\
\hline
\end{tabular}

\section{B. Overall results}

Many apps in the sampled dataset of 100,000 apps do not use the top 10 API methods. Thus, we immediately filter such apps from the study.Besides, ParamHarver fails on some apps due to time/memory constraints.As a result, 22,681 apps are considered for further analysis.

Fig. 2 plots the distribution of the number of top-10 API methods used by the examined Android apps. The median and mean value are 2 and 3.56 respectively. We further perform a correlation study (through Spearman's rho) between the number of API methods and the size of code in apps (i.e., the size of DEX file). The spearman correlation results $($ rho $=-0.0046, p-$ value $>0.1)$ indicates that these two variables are not significantly correlated.

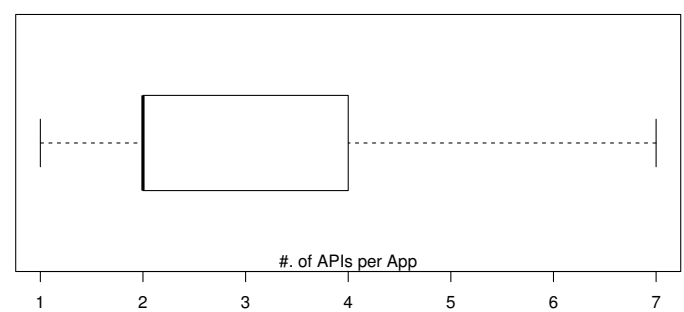

Fig. 2: The number of API methods per app without considering outliers.

\section{Duplication of Parameter Values}

Table II illustrates the overall results of our study. The second column indicates the position of the considered parameters for a given API, e.g., 1 means the first parameter. The third and fourth column shows the number of values harvested by ParamHarver, where (T) means the total number of successfully harvested values (excluding (.*), NULL-CONSTANT and empty results) while (D) means the number of distinct values.

The huge difference between the third and fourth column suggests that most parameter values are used in duplicate. This is insightful, as most parameter values are independently used
TABLE II: The investigation results.

\begin{tabular}{lcrr}
\hline Method Name & Parameter Seq & Count (T) & Count (D) \\
\hline getLastKnownLocation & 1 & 32,832 & 3 \\
requestLocationUpdates & 1 & 11,691 & 5 \\
isProviderEnabled & 1 & 7,985 & 6 \\
putString & 2 & 1,330 & 33 \\
& 3 & 185 & 11 \\
sendTextMessage & 1 & 344 & 65 \\
getAccountsByType & 3 & 6,909 & 150 \\
putInt & 1 & 2,034 & 8 \\
restartPackage & 1 & 1,666 & 24 \\
setVideoPath & 1 & 187 & 48 \\
getProvider & 1 & 22 & 8 \\
\hline
\end{tabular}

by many different apps, we would expect that this situation happens only in API methods whose parameter values are defined in advance, e.g., getLastKnownLocation(). However, we would not think it likely that this also happens in other API methods whose parameter values are strongly correlated to developers' requirements. Let us take sendTextMessage() as an example, it has two parameters (the first one indicates the target phone number and the third one indicates the message context) that are totally correlated to developers' requirements. In our observation, phone number 106909990999, as the first parameter, has been used 43 times. message cxye, as the third parameter, has been used 127 times.

We further investigate the reason why 106909990999 is used as a target phone number by 43 apps to send SMS. Interestingly, among the 43 apps, we collect seven distinct package names, meaning most of them are actually variants of same apps. Even there are seven distinct package names, all of them are signed by one of two distinct certificates. Additionally, those 43 apps shared the same prefix (com.feedov) for their package names. Based on these evidences, we can observe that duplicated parameter values could be leveraged to find app variants. The investigation results on message cxye has also confirmed this finding.

\section{Recommendation of Parameter Values}

Table III comparatively summarizes the parameter values that are harvested by ParamHarver on the four methods of class LocationManager. The three most used values (in gray) are powered by the basic Android SDK while the remaining values are powered by third-party services. The most used values, without any doubt, are "gps" and "network". "go2map" is the least used value, which is used only one time.

Being the fact that parameter values in Android API methods are usually used in duplicate, we believe our approach could be leveraged to recommend possible parameter values for API methods. Recall that in the introduction we have shown the insufficient documentation of Android API methods, where it is difficult to know what should be the parameter value of the API method getLastKnownLocation(). Now, with our approach, we could give a hint to developers that the first three values ("gps", "network" and "passive") are relevant to their needs, although the other four values are also possible. Furthermore, "gps" or "network" are even more applicable than "passive", as indicated by the huge difference of used times among them. 
TABLE III: Comparative results of LocationManager-related API methods.

\begin{tabular}{l|ccc|ccccc}
\hline Method Name & gps & network & passive & lbs & go2map & MapABCNetwork & AutonavicellLocationProvider & Total \\
\hline getLastKnownLocation & 15,263 & 17,550 & 19 & 0 & 0 & 0 & 0 \\
requestLocationUpdates & 3,714 & 7,916 & 9 & 51 & 1 & 0 & 32,832 \\
isProviderEnabled & 6,469 & 1,494 & 1 & 17 & 0 & 1 & 1,691 \\
getProvider & 53 & 56 & 0 & 6 & 0 & 3 & 3 \\
\hline Total & 25,499 & 27,016 & 29 & 74 & 1 & 4 & 9 \\
\hline
\end{tabular}

TABLE IV: The harvested phone numbers and their status $(\mathrm{C}=$ Count, $S=$ Status, $\checkmark=$ premium, $X=$ non-premium, $?=$ unknown).

\begin{tabular}{|c|c|c|c|c|c|}
\hline Number & $\mathrm{C}$ & $\mathrm{S}$ & Number & $\mathrm{C}$ & $\mathrm{S}$ \\
\hline 10086 & 129 & $x$ & 1066185829 & 2 & $\sqrt{ }$ \\
\hline 106909990999 & 43 & $\checkmark$ & 1066953930 & 2 & $\checkmark$ \\
\hline 10659811002 & 14 & $\sqrt{ }$ & 3354 & 1 & $?$ \\
\hline 10001 & 13 & $x$ & 3353 & 1 & $?$ \\
\hline 7132 & 12 & ? & 18703750375 & 1 & $x$ \\
\hline 12114 & 10 & $x$ & 15919831203 & 1 & $x$ \\
\hline 10010 & 7 & $x$ & 15860282110 & 1 & $x$ \\
\hline 1065515810002 & 6 & $\checkmark$ & +19494368398 & 1 & $?$ \\
\hline 106588003013891 & 6 & $\checkmark$ & 0 & 1 & $?$ \\
\hline 1065889915 & 6 & $\checkmark$ & 04800 & 1 & $?$ \\
\hline 13811558614 & 6 & $x$ & 10086999 & 1 & $x$ \\
\hline 15919479044 & 6 & $x$ & $1065-5021-80133$ & 1 & $\checkmark$ \\
\hline 1065843601 & 5 & $\checkmark$ & $1065-71090-88877$ & 1 & $\checkmark$ \\
\hline 1065880004 & 3 & $\checkmark$ & $1065-9020-5111-191$ & 1 & $\checkmark$ \\
\hline 10660596 & 3 & $\checkmark$ & 1065505796084 & 1 & $\checkmark$ \\
\hline 1066156686 & 3 & $\checkmark$ & 10655133 & 1 & $\checkmark$ \\
\hline 13701370135 & 3 & $x$ & 10655576 & 1 & $\checkmark$ \\
\hline 13720000800 & 3 & $x$ & 10657525748900014 & 1 & $\checkmark$ \\
\hline 13720062005 & 3 & $x$ & 106580808 & 1 & $\checkmark$ \\
\hline 13720072005 & 3 & $x$ & 10658422 & 1 & $\checkmark$ \\
\hline 1065502182177 & 2 & $\checkmark$ & 10659057110094 & 1 & $\checkmark$ \\
\hline 10657109050762 & 2 & $\checkmark$ & 1066017801 & 1 & $\checkmark$ \\
\hline 10658368 & 2 & $\checkmark$ & 10669079 & 1 & $\checkmark$ \\
\hline 10658424 & 2 & $\checkmark$ & 123987651017 & 1 & ? \\
\hline 1065902163958 & 2 & $\checkmark$ & 13564332483 & 1 & $x$ \\
\hline 106601412004 & 2 & $\checkmark$ & 13640534425 & 1 & $x$ \\
\hline 1066057101 & 2 & $\checkmark$ & 15239463832 & 1 & $x$ \\
\hline 1066057103 & 2 & $\checkmark$ & 15859268161 & 1 & $x$ \\
\hline 15645027999 & 2 & $x$ & 400 & 1 & $x$ \\
\hline 13823308135 & 2 & $x$ & 4860008 & 1 & $?$ \\
\hline 13646870394 & 2 & $x$ & 7838613121 & 1 & $?$ \\
\hline 106901952345 & 2 & $\checkmark$ & 9494367679 & 1 & ? \\
\hline 1069003801012038 & 2 & $\checkmark$ & & & \\
\hline
\end{tabular}

\section{E. Parameter Values for Malware Identification}

As indicated by [11], some sensitive runtime data (or parameter values of API methods) in Android apps can actually be used for identifying malicious apps. Indeed, as shown in Section III-C, many sensitive parameter values are actually used in many samples, making them good candidates for blacklisting.

Let us consider method sendTextMessage() as an example. Malicious apps could use it to silently send SMS to premium numbers, causing a high financial harm to users. In our investigation, we have harvested 65 phone numbers, which are listed in Table IV To verify whether our findings are able to identify other malicious apps, we take the list of numbers in Table IV as a blacklist to evaluate the MalGenome project [13]. With this simple setting, we are able to flag 158 (out of 1,260) apps as malicious. Table $\mathrm{V}$ illustrates more details on our findings, where we classify our findings through their family labels. Interestingly, only based on a blacklist of phone numbers, we are able to flag malicious apps from 15 families. Additionally, all apps of 8 families (out of 15) are
TABLE V: Malware identification results.

\begin{tabular}{lcl}
\hline Family & Count & Numbers \\
\hline DroidDream & $2 / 16$ & 10001 \\
YZHC & $\mathbf{2 2 / 2 2}$ & 10086 \\
zHash & $\mathbf{1 1 / 1 1}$ & $10655133,10001,10086,10010$ \\
Geinimi & $5 / 69$ & $3353,10001,13564332483$ \\
FakePlayer & $\mathbf{6 / 6}$ & $7132,3353,3354$ \\
Asroot & $1 / 8$ & 10001 \\
BaseBridge & $46 / 122$ & 10001,10086 \\
BeanBot & $\mathbf{8 / 8}$ & 10086 \\
Pjapps & $21 / 58$ & $10086999,10086,10010$ \\
HippoSMS & $\mathbf{4 / 4}$ & 1066156686 \\
Bgserv & $\mathbf{9 / 9}$ & 10086,10010 \\
DroidDreamLight & $1 / 46$ & 10086,10010 \\
RogueSPPush & $\mathbf{9 / 9}$ & 10086 \\
Zsone & $\mathbf{1 2 / 1 2}$ & $10655133,1066953930,106601412004$, \\
NickySpy & $1 / 2$ & $1066185829,10086,10010$ \\
& & 15859268161 \\
\hline
\end{tabular}

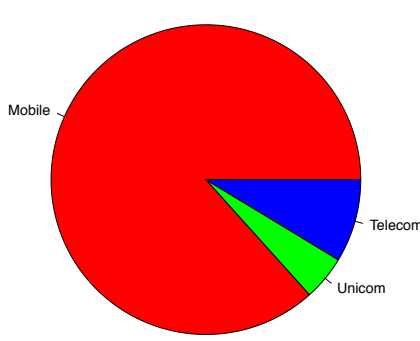

(a) Normal number.

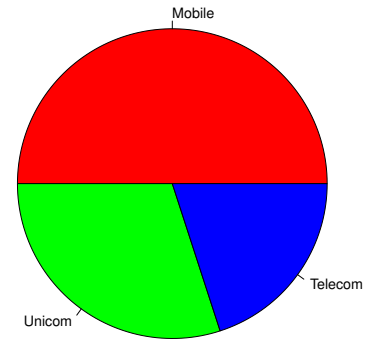

(b) Premium number.
Fig. 3: Distribution of the usage of phone numbers in different service providers of China.

fully identified.

\section{F. Parameters for Trend Analysis}

After harvesting a large set of parameter values, we could based on them to perform trend analysis. we now show an example of trend analysis that we observed through the harvested phone numbers.

In china, mainly, there are three service providers $(\mathrm{SP})^{4}$ 1) China Mobile, whose normal service numbers start from 10086, while premium numbers start from 10657 or 10658 ; 2) China Telecom, whose normal service numbers start from 10001 while premium numbers start from 10659; and 3) China Unicom, whose normal service numbers start from 10010 while premium numbers start from 106555. Based on these information, we manually evaluated the harvested numbers (cf. Table IV and classified them into three categories: 1) known

\footnotetext{
${ }^{4}$ https://en.wikipedia.org/wiki/Telecommunications_industry_in_China.

${ }^{5}$ http://www.miit.gov.cn/n11293472/n11293832/n11293907/n11368223/ n12977312.files/n12977310.doc
} 
premium numbers $(\checkmark) ; 2)$ known non-premium numbers $(X)$, note that these numbers however could also be used for malicious purpose as all of them are harvested from malicious apps; and 3) unknown numbers (?), for which their statuses need to be further investigated.

Fig. 3 presents the distribution of the use of service numbers in different SPs: the normal service is in Fig. 3a while the premium service is in Fig. 3b. Both figures have shown that there are more malicious apps that target China Mobile than the other two SPs, suggesting that China Mobile occupies more marketing shares than the other SPs. This trend is actually reflecting the real situation, where China Mobile is currently dominating the Chinese SP market shares. Finally, we note that both Telecom and Unicorn account for less than $25 \%$ for normal numbers but increase to over $50 \%$ for premium numbers. This result suggests that malware writers tend to diversify their SP targets.

\section{G. Threats to Validity}

At the moment, our approach is unaware of obfuscation, which may lead to incomplete results. Like many other static analysis approaches, our approach is unaware of native code, multi-threads and reflections. Besides, our static analysis is also unaware of the inter-component communication (ICC) mechanism. Other approaches could be leveraged to mitigate these kinds of threats (e.g., IccTA [4] for the ICC mechanism). So far, we perform string analysis for parameters, in our future work, we plan to extend this work to also support the analysis of object parameters.

\section{RELATED WORK}

There are several approaches closing to our work. One of the most sophisticated ones is Harvester [11], which is dedicated to extract runtime values from any position in the Android code. Although it is not the focus, Harvester could be also leveraged to extract the parameter values from all the Android API methods in Android apps. However, the implementation between Harvester and our tool ParamHarver is quite different. As Harvester needs to execute the apps while ParamHarver is a pure static approach which does not need to really launch the apps.

Another related work is Precise [12], which recommends parameters of API methods automatically so as to ease the use of API methods, as the API documentations are usually incomplete. Precise mines existing code base (through certain scenario) to build a parameter usage database and then based on it to provide on-demand queries for parameter candidates through concrete usage patterns. Our work can be taken as a simplified version of Precise, based on the harvested practical parameters, we are also able to recommend the appropriate parameter candidates.

There are a batch of works that investigate the Android API methods [6]-[8] or libraries [5]. Although those works are not focusing on the parameters of API methods, we believe that our findings (the harvested parameter values) can be useful for them. For example, a suddenly increase of parameter values (starting from a specific API version) would suggest that the API or library is probably updated (e.g., to provide more functions).

\section{CONCLUSiOn AND FUture WORKS}

In this paper, we have first presented a tool called ParamHarver to automatically harvest parameter values of Android API methods. Then, we launched ParamHarver on a set of 100,000 apps with 10 top used Android API methods. In the next step, we perform a preliminary investigation on what we can gain from the harvested parameter values, from which we show that 1) parameter values could be leveraged to find app variants; 2) parameter values harvested from a large set of apps could be used to recommend appropriate values for practical use of API methods; 3) parameter values of some sensitive API methods can be used to identify malicious apps; and 4) parameter values, to some extend, can be leveraged to perform trend analysis.

\section{ACKNOWLEDGMENTS}

This work was supported by the Fonds National de la Recherche (FNR), Luxembourg, under the project AndroMap C13/IS/5921289.

\section{REFERENCES}

[1] Kevin Allix, Quentin Jérome, Tegawende F Bissyandé, Jacques Klein, Radu State, and Yves Le Traon. A forensic analysis of android malwarehow is malware written and how it could be detected? In COMPSAC'14.

[2] Steven Arzt, Siegfried Rasthofer, Christian Fritz, Eric Bodden, Alexandre Bartel, Jacques Klein, Yves Le Traon, Damien Octeau, and Patrick McDaniel. Flowdroid: Precise context, flow, field, object-sensitive and lifecycle-aware taint analysis for android apps. In PLDI, 2014.

[3] Li Li, Kevin Allix, Daoyuan Li, Alexandre Bartel, Tegawendé F Bissyandé, and Jacques Klein. Potential Component Leaks in Android Apps: An Investigation into a new Feature Set for Malware Detection. In $Q R S, 2015$.

[4] Li Li, Alexandre Bartel, Tegawendé F Bissyandé, Jacques Klein, Yves Le Traon, Steven Arzt, Siegfried Rasthofer, Eric Bodden, Damien Octeau, and Patrick Mcdaniel. IccTA: Detecting Inter-Component Privacy Leaks in Android Apps. In ICSE, 2015.

[5] Li Li, Tegawendé F Bissyandé, Jacques Klein, and Yves Le Traon. An Investigation into the Use of Common Libraries in Android Apps. In SANER, 2016.

[6] Mario Linares-Vásquez, Gabriele Bavota, Carlos Bernal-Cárdenas, Massimiliano Di Penta, Rocco Oliveto, and Denys Poshyvanyk. Api change and fault proneness: a threat to the success of android apps. In FSE'13.

[7] Mario Linares-Vásquez, Gabriele Bavota, Massimiliano Di Penta, Rocco Oliveto, and Denys Poshyvanyk. How do api changes trigger stack overflow discussions? a study on the android sdk. In ICPC, 2014.

[8] Tyler McDonnell, Bonnie Ray, and Miryung Kim. An empirical study of api stability and adoption in the android ecosystem. In ICSM, 2013.

[9] Damien Octeau, Somesh Jha, Matthew Dering, Patrick Mcdaniel, Alexandre Bartel, Li Li, Jacques Klein, and Yves Le Traon. Combining static analysis with probabilistic models to enable market-scale android inter-component analysis. In POPL, 2016.

[10] Damien Octeau, Daniel Luchaup, Matthew Dering, Somesh Jha, and Patrick McDaniel. Composite constant propagation: Application to android inter-component communication analysis. In ICSE, 2015.

[11] Siegfried Rasthofer, Steven Arzt, Marc Miltenberger, and Eric Bodden. Harvesting runtime values in android applications that feature antianalysis techniques. In NDSS, 2016.

[12] Cheng Zhang, Juyuan Yang, Yi Zhang, Jing Fan, Xin Zhang, Jianjun Zhao, and Peizhao Ou. Automatic parameter recommendation for practical api usage. In ICSE, 2012.

[13] Yajin Zhou and Xuxian Jiang. Dissecting android malware: Characterization and evolution. In $S \& P, 2012$ 